Journal of Animal and Veterinary Advances $10(1): 106-110,2011$

ISSN: $1680-5593$

(C) Medwell Journals, 2011

\title{
Prevalence of net $B$ Gene among Clostridium perfringens Isolates Obtained from Healthy and Diseased Chickens
}

\author{
A. Tolooe, B. Shojadoost, S.M. Peighambari and Y. Tamaddon \\ Department of Clinical Sciences, Faculty of Veterinary Medicine, \\ University of Tehran, P.O. Box 14155-6453, Tehran, Iran
}

\begin{abstract}
Necrotic Enteritis (NE) is the most clinically dramatic bacterial enteric disease of poultry induced by C. perfringens that affects industrial poultry worldwide. C. perfringens produces a large range of potent toxins that are responsible for severe diseases in humans and animals. The critical importance of netB, the recently identified pore-forming toxin for the development of $\mathrm{NE}$ is still under discussion. In this study C. perfringens isolates from healthy and diseased poultry flocks were analyzed by PCR in order to determine the presence of The netB for the first time in Asia. The netB was detected in $52.8 \%$ isolates from diseased flocks but not in any isolates from healthy ones. The products of four positive netB PCR reactions were sequenced. Comparison of the isolates sequences revealed $100 \%$ identity to each other and to the netB sequences available in GenBank. netB can not be the only $C$. perfringens virulence factor involved in $\mathrm{NE}$, since not all $C$. perfringens isolates from birds associated with $\mathrm{NE}$ contain the netB gene. However, further investigations are required to determine the role of netB in development of NE.
\end{abstract}

Key words: Clostridium perfringens, necrotic enteritis, toxin, netB, chicken, isolates sequences

\section{INTRODUCTION}

Among pathogenic bacteria, different species of the Clostridia genus produce the most variable type of toxins which are considered as the main virulence determinants of these bacteria (Popoff and Bouvet, 2009). Clostridium perfringens, an anaerobic gram-positive sporeforming bacterium is ubiquitous in the intestinal flora of human and animals and known to be the most widely distributed pathogen in nature. C. perfringens produce a large range of potent toxins and enzymes that are responsible for severe diseases in humans and other animals (Popoff and Bouvet, 2009). In the last decade, a great effort has been made to understand the mechanism of action, structure and function of these toxins.

Some toxins are known as potent virulence factors while the implication of other toxins in pathogenicity is questionable (Rood, 1998). In a commonly used classification scheme, C. perfringens is divided into five toxinotypes (A-E) based on the production of four major toxins (alpha, beta, epsilon and iota) where each type carries a different combination of the toxin genes however, this bacterium also produces at least 13 other toxins such as C. perfringens Enterotoxin (CPE), theta toxin, beta2 toxin and netB toxin (Keyburn et al., 2008; Petit et al., 1999). Specific C. perfringens toxin types are associated with particular human and animal diseases. C. perfringens is often found in the intestinal tract of healthy birds but it can cause outbreaks of disease in many species of poultry and especially in broiler and turkey flocks (Songer, 1996). Clostridiosis occurs both as an acute clinical disease called as Necrotic Enteritis (NE) causing high mortality and as a subclinical disease with focal necrosis in the intestine (subclinical NE) or C. perfringens-associated hepatitis with cholangiohepatitis or fibrinoid necrosis in the liver (Cooper and Songer, 2009). NE is the most clinically dramatic bacterial enteric disease of poultry induced by $C$. perfringens which affects industrial poultry worldwide and is a global problem (Cooper and Songer, 2009). Much of the current research on $\mathrm{NE}$ has focused on finding a definitive toxin that is responsible for causing disease. The majority of C. perfringens isolates from poultry belong to toxin type A but a few belong to type C (Cooper and Songer, 2009). The majority of the chicken strains are toxinotype A, meaning that they carry the $c p a$ gene encoding alpha toxin (Petit et al., 1999). For a long time, it was believed that this alpha toxin was the major virulence factor involved in NE (Fukata et al., 1988). Recently, the role of C. perfringens alpha toxin in NE is disputed. A cpa knockout mutant from a virulent $C$. perfringens chicken strain was still capable of inducing necrotic lesions in the

Corresponding Author: Bahram Shojadoost, Department of Clinical Sciences, Faculty of Veterinary Medicine, University of Tehran, P.O. Box 14155-6453, Tehran, Iran 
gut of experimentally infected broilers (Keyburn et al., 2006). In addition, almost 2 years ago, the netB toxin and its encoding gene (netB) were first identified in an Australian strain of $C$. perfringens type $\mathrm{A}$ that was isolated from a chicken suffering from NE (Keyburn et al., 2008). Its importance in NE was shown when a netB mutant $C$. perfringens strain did not cause NE in an experimental chicken model and virulence was restored when a functional netB gene was introduced back into the mutant strain (Keyburn et al., 2008).

The critical importance of netB for the development of necrotic enteritis is still under discussion as occasionally isolates that lack the netB gene can be found in birds suffering from NE and NE has been reproduced with netB negative isolates (Abildgaard et al., 2010; Cooper and Songer, 2010). However, the exact role of this toxin in pathogenicity of NE still needs to be elucidated. Various epidemiological studies of C. perfringens strains from varied geographical locations have been published but very few studies from Asian countries have been published on $C$. perfringens-induced NE in poultry and very little knowledge of the $C$. perfringens genetic profile in Asia is available. In the present study, for the first time in Asia, we analyzed the C. perfringens isolates from poultry flocks in a single PCR assay in order to determine the presence of netB gene and examined its occurrence with respect to $\mathrm{NE}$ in chickens.

\section{MATERIALS AND METHODS}

Bacterial isolates and bacteriological procedures: About 79 isolates of $C$. perfringens type A collected during the period of 2005-2008 and kept in the laboratory in 50\% glycerol at $-70^{\circ} \mathrm{C}$ were used for this study. The collection consisted of 36 isolates obtained from six NE-positive flocks (broiler) and 43 strains obtained from four NE-negative flocks (two broiler, one layer and one broiler breeder). Toxinotypes of $C$. perfringens isolates were determined by Multiplex PCR in the previous study. The frozen C. perfringens isolates were cultivated in Brain Heart Infusion (BHI) and incubated anaerobically at $37^{\circ} \mathrm{C}$ for 24-36 h.

Samples were sub-cultured anaerobically in blood agar plates containing 7\% defibrinated sheep blood, Tryptose Sulfite Cycloserine agar (TSC) and Tryptose Sulfite Neomycin agar (TSN). The identity of the isolates was confirmed by characteristic colony morphology, hemolytic pattern, gram staining and biochemical tests as previously described (Quinn et al., 1994). All culture media and additives used in this study were provided from Merck (Germany). Due to recent discovery of netB gene, there was no netB-positive standard strain of $C$. perfringens for using in PCR. However, the first identified netB-positive isolate was kept and used in all $P C R$ reaction sets.

Single PCR for $\boldsymbol{n e t} B$ gene: To extract bacterial DNA, a single colony of each $C$. perfringens isolate grown on blood agar plate for overnight at $37^{\circ} \mathrm{C}$ suspended into $100 \mu \mathrm{L}$ distilled water in a clean $1.5 \mathrm{~mL}$ microtube, boiled for $10 \mathrm{~min}$ and centrifuged for $10 \mathrm{~min}$ at $10000 \times \mathrm{g}$. The supernatants were carefully removed and used as template DNA. The concentration of DNA was determined by Biophotometer (Eppendorff, Germany) and adjusted to approximately $50 \mathrm{ng}$ for each $\mathrm{PCR}$ reaction. To detect netB gene previously developed forward (5' GCTGGTGCTGGAATAAATGC-3') and reverse (5'TCGCCATTGAGTAGTTTCCC-3') primers were used (Keyburn et al., 2008). Amplification reactions were carried out in a $25 \mu \mathrm{L}$ reaction volume containing: $2 \mu \mathrm{L}$ $10 \times$ PCR buffer, $2.5 \mathrm{mM} \mathrm{MgCl}_{2}, 0.2 \mathrm{mM}$ dNTPs mixture, 2.5 units of Taq DNA polymerase, $0.1 \mu \mathrm{M}$ of each primers, $\mathrm{dH}_{2} \mathrm{O}$ and $5 \mu \mathrm{L}$ of template DNA solution. Negative and positive controls were included in all PCR reaction sets. Amplification was programmed in a thermocycler (Gradient Mastercycler, Eppendorff, Germany) as follows: $94^{\circ} \mathrm{C}$ for $2 \mathrm{~min}$ followed by 35 cycles of $94^{\circ} \mathrm{C}$ for $30 \mathrm{sec}$, $55^{\circ} \mathrm{C}$ for $30 \mathrm{sec}, 72^{\circ} \mathrm{C}$ for $60 \mathrm{sec}$ and a final extension at $72^{\circ} \mathrm{C}$ for $12 \mathrm{~min}$ (Keyburn et al., 2008). The amplification products were detected by gel electrophoresis (Apelex, France) in $1.5 \%$ agarose gel in $1 \times \mathrm{TAE}$ buffer, stained with $0.5 \mu \mathrm{g} \mathrm{mL}{ }^{-1} \mathrm{EtBr}$. Amplified bands were visualized and photographed under UV transillumination (UVP Visi-DocIt System, UK). The primers and other materials used in $\mathrm{PCR}$ reaction were provided by Cinnagen (Tehran, Iran).

DNA sequencing of netB PCR products: Four $C$. perfringens isolates (ATBS61IR, ATBS65IR, ATBS94IR and ATBS120IR) obtained from separate diseased flocks were selected and their relevant PCR amplified products for netB were purified using the Gene JET ${ }^{\mathrm{TM}}$ Gel Extraction Kit (Fermentas Life Science, Germany) according to the manufacturer's instructions and submitted for automated sequencing in both directions at the Geneservice ${ }^{\mathrm{TM}}$, Source BioScience (Cambridge, England) using PCR primers as sequencing primers. The sequence data were submitted to GenBank under the accession numbers GU453172 and GU581173-5.

\section{RESULTS AND DISCUSSION}

Single PCR for net $B$ gene: The presence of the netB gene was examined in all isolates by single PCR (Fig. 1). The netB gene was detected in 19/36(52.8\%) isolates from diseased flocks but not in any isolates from healthy 


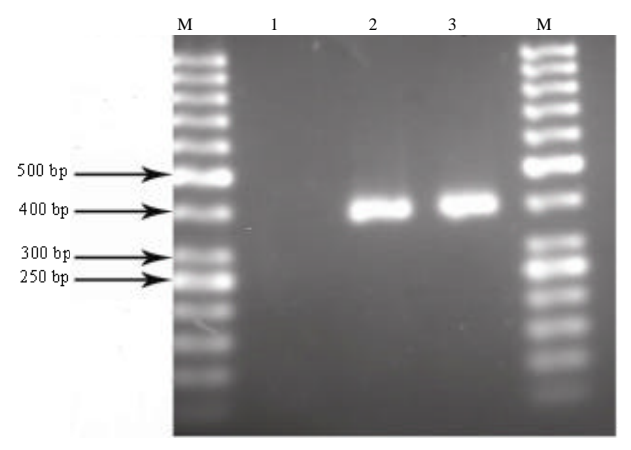

Fig. 1: Agarose gel (1.5\%) electrophoresis results of the PCR assay for the detection of a $384 \mathrm{bp}$ fragment of the netB gene in C. perfringens. Lanes M: Size marker (GeneRuler 50 bp DNA Ladder, Fermentas); Lane 1: Negative control; Lanes 2-3: netB-positive C. perfringens isolates from birds suffering from NE

flocks. All isolates from a single flock showed an identical pattern except in flock no. 4 in which one isolate was negative for $n e t B$ gene but the rest were positive (Table 1).

Sequence analysis of netB PCR products: Comparison of four Iranian C. perfringens isolates sequences by Blastn (http://www.ncbi.nlm.nih.gov/Blast) at the nucleotide level revealed $100 \%$ identity to each other and to the netB sequences of $C$. perfringens strains available in GenBank.

The recently discovered net $B$ toxin gene was exclusively found in NE-associated flocks in the study although, all C. perfringens isolates from three NEpositive flocks were netB negative (Table 1).

Since the discovery of netB in 2008 , the presence of the netB gene have been screened among a wide variety of C. perfringens isolates from Australia, Canada, United States and some European countries (Chalmers et al., 2008; Johansson et al., 2010; Keyburn et al., 2009; Martin and Smyth, 2009). However, this study for the first time reports the presence of $n e t B$ gene among C. perfringens isolates from Asia. Similar to the findings, the netB gene has not been detected in all isolates from definitive cases of NE (Abildgaard et al., 2010; Chalmers et al., 2008; Johansson et al., 2010; Keyburn et al., 2009; Martin and Smyth, 2009).

Despite the first report on the detection of netB gene among isolates from diseased chickens, the presence of this gene among $C$. perfringens isolates from healthy chickens was also confirmed in later studies (Abildgaard et al., 2010; Chalmers et al., 2008; Johansson et al., 2010; Keyburn et al., 2008, 2009;

\begin{tabular}{llllll}
\multicolumn{5}{l}{ Table 1: Characteristics of Clostridium } & perfringens \\
\hline $\begin{array}{l}\text { Flock } \\
\text { no. }\end{array}$ & $\begin{array}{l}\text { Flock } \\
\text { status }\end{array}$ & $\begin{array}{l}\text { Flock } \\
\text { type }\end{array}$ & $\begin{array}{l}\text { No. of } \\
\text { isolates }\end{array}$ & $\begin{array}{l}\text { Toxin } \\
\text { types }\end{array}$ & netB \\
\hline 1 & Diseased & Broiler & 4 & Type A & - \\
2 & Diseased & Broiler & 8 & Type A & + \\
3 & Diseased & Broiler & 4 & Type A & - \\
4 & Diseased & Broiler & 7 & Type A & $6+/ 1-$ \\
5 & Diseased & Broiler & 8 & Type A & - \\
6 & Diseased & Broiler & 5 & Type A & + \\
7 & Healthy & Broiler & 25 & Type A & - \\
8 & Healthy & Broiler & 4 & Type A & - \\
9 & Healthy & Layer & 10 & Type A & - \\
10 & Healthy & Breeder & 4 & Type A & \\
\hline
\end{tabular}

Martin and Smyth, 2009). Chalmers et al. (2008) found the presence of netB gene only in isolates associated with NE outbreaks in Canada but not in any isolates from healthy birds which correspond with the findings in this study. In another North American survey, it was found that the majority of $C$. perfringens isolates from chickens with clinical signs of NE carried the netB gene (58.3\%) whereas only a small percentage $(8.6 \%)$ of isolates from healthy birds carried this gene (Martin and Smyth, 2009). The highest percentage (>90\%) of netB gene positive among $C$. perfringens isolates from diseased chickens was reported in a single broiler flock in Sweden. However, in the same flock, $25 \%$ of the isolates from apparently healthy birds were also netB positive (Johansson et al., 2010). Possibly, the reason for the detection of a high percentage of net $B$ positive cases was that all isolates were from a single flock which represents the dominant $C$. perfringens population in the chicken organ lesions. Recently, Keyburn et al. (2009) studied various $C$. perfringens isolates from NE-positive and NE-negative flocks from three continents and found $70 \%(31 / 44)$ and $3.6 \%(2 / 55)$ netB positive isolates, respectively. In an Italian study, 107 C. perfringens isolates were tested for the presence of netB gene and it was found that $27 \%$ $(29 / 107)$ of isolates were netB positive in which $93 \%$ $(27 / 29)$ of isolates had been were originated from birds affected by NE and other intestinal disorders (Drigo et al., 2009). A recent Danish study showed a prevalence of approximately 50 and $60 \%$ of netB gene among isolates from NE-associated and healthy flocks, respectively (Abildgaard et al., 2010). The Danish study was the first and the only one that reported the presence of netB gene in isolates from healthy chickens more than that of in isolates from NE-positive chickens.

The presence of netB gene has also been shown in one non-poultry related C. perfringens isolate. Martin and Smyth (2009) reported isolation of the first netB-positive C. perfringens from the liver abscesses in a cow died with gastrointestinal disease. This non-poultry isolate was shown to cause lesions characteristic of avian NE in a chicken disease model (Smyth and Martin, 2010). This 
finding was in contrast to another recent study which demonstrated the failure of a non-avian netB-positive C. perfringens isolate to effectively colonize birds following challenge with a high titre of infective inoculum. It was suggested that the overall deficiency in poultry colonization factors may be the reason for this failure (Cooper et al., 2010).

The presence of toxin genes in C. perfringens isolates does not solely determine the clinical importance of the isolates and there are some predisposing factors that have been associated with the selection of toxigenic $C$. perfringens and consequently, the development of disease. Not all C. perfringens strains isolated from birds that clearly displayed signs of necrotic enteritis were netB positive and in one particular study two $C$. perfringens isolates that were negative for $n e t B$ gene produced $\mathrm{NE}$ in 6.7 and $16.7 \%$ of inoculated birds (Cooper and Songer, 2010). These results imply that although there is a clear association between the presence of netB toxin gene and development of NE, there may be other (yet to be determined) virulence factors that are produced by these netB-negative disease-producing isolates. While it is possible that NE may results from the interaction of several toxic molecules, further investigation on this issue appears to be necessary. It is noteworthy that all netBpositive isolates identified in the study were cpb2positive too. A recent study has revealed that in several NE-positive isolates the netB gene is part of a large potential pathogenicity locus (Lepp et al., 2010). The identification of pathogenicity loci may lead to the discovery of additional virulence factors that are involved in the pathogenesis of disease (Lepp et al., 2010).

\section{CONCLUSION}

This study demonstrated the presence of netB gene in more than half of the isolates from diseased flocks but not in any isolates from healthy flocks. However, netB can not be the only $C$. perfringens virulence factor involved in avian NE, since not all $C$. perfringens isolates from birds with NE contain the netB gene. According to the information obtained from the study and other investigations, netB toxin may not be an obligate requirement for poultry $C$. perfringens virulence and at least the presence of netB may not be essential for the disease process in all C. perfringens isolates. Although, the role of netB in the induction of NE needs further investigation. In addition, comparison of four Iranian C. perfringens isolates sequences revealed $100 \%$ identity to each other and to the netB sequences of $C$. perfringens strains available in GenBank.

Future investigations should focus on the ability of toxin production by this gene, experimental studies in a disease model to investigate the disease producing capabilities of the netB positive and negative strains recovered from cases of $\mathrm{NE}$ and also the netB negative strains recovered from healthy chickens, vaccination with various toxoids and subsequent challenge for opening significant opportunities for the development of novel vaccines against $\mathrm{NE}$ in poultry, study on the regulatory mechanisms involved in the expression of netB toxin, identification the netB in $C$. perfringens isolates from species other than chicken, considering the roles of external and predisposing factors in pathogenesis of $\mathrm{NE}$ and identification new genes for toxins or related enzymes in future as was as the case for C. perfringens netB.

\section{ACKNOWLEDGEMENTS}

This research was funded by a grant $(7508049 / 6 / 7)$ from the Research Council of the University of Tehran. The researchers have special thanks to Prof. J. Glenn Songer, Department of Veterinary Science and Microbiology, The University of Arizona for his helpful suggestions and comments at different stages of this research.

\section{REFERENCES}

Abildgaard, L., T.E. Sondergaard, R.M. Engberg, A. Schramm and O. Hojberg, 2010. In vitro production of necrotic enteritis toxin $B, N e t B$, by netB-positive and netB-negative Clostridium perfringens originating from healthy and diseased broiler chickens. Vet. Microbiol., 144: 231-235.

Chalmers, G., H.L. Bruce, D.B. Hunter, V.R. Parreira and R.R. Kulkarni et al., 2008. Multilocus sequence typing analysis of Clostridium perfringens isolates from necrotic enteritis outbreaks in broiler chicken populations. J. Clin. Microbiol., 46: 3957-3964.

Cooper, K.K. and J.G. Songer, 2009. Necrotic enteritis in chickens: A paradigm of enteric infection by Clostridium perfringens type A. Anaerobe, 15: 55-60.

Cooper, K.K. and J.G. Songer, 2010. Virulence of Clostridium perfringens in an experimental model of poultry necrotic enteritis. Vet. Microbiol., 142: $323-328$.

Cooper, K.K., J.R. Theoret, B.A. Stewart, H.T. Trinh, R.D. Glock and J.G. Songer, 2010. Virulence for chickens of Clostridium perfringens isolated from poultry and other sources. Anaerobe, 16: 289-292.

Drigo, I., F. Agnoletti, C. Bacchin, A. Guolo, M. Cocchi, M. Bonci and L. Bano, 2009. Diffusion of Clostridium perfringens NetB positive strains in healthy and diseased chickens. Ital. J. Anim. Sci., 8: 761-764.

Fukata, T., Y. Hadate, E. Baba, T. Uemura and A. Arakawa, 1988. Influence of Clostridium perfringens and its toxin in germ-free chickens. Res. Vet. Sci., 44: 68-70. 
Johansson, A., A. Aspan, M. Kaldhusdal and B.E. Engstrom, 2010. Genetic diversity and prevalence of netB in Clostridium perfringens isolated from a broiler flock affected by mild necrotic enteritis. Vet. Microbiol., 144: 87-92.

Keyburn, A.L., J.D. Boyce, P. Vaz, T.L. Bannam and M.E. Ford et al., 2008. NetB, a new toxin that is associated with avian necrotic enteritis caused by Clostridium perfringens. PLOS Pathog., 4: e26-e26.

Keyburn, A.L., S.A. Sheedy, M.E. Ford, M.M. Williamson, M.M. Awad, J.I. Rood and R.J. Moore, 2006. Alpha-toxin of Clostridium perfringens is not an essential virulence factor in necrotic enteritis in chickens. Infect. Immun., 74: 6496-6500.

Keyburn, A.L., X.X. Yan, T.L. Bannam, F. Van Immerseel, J.I. Rood and R.J. Moore, 2009. Association between avian necrotic enteritis and Clostridium perfringens strains expressing NetB toxin. Vet. Res., 41: 21-21.

Lepp, D., B. Roxas, V.R. Parreira, P.R. Marri and E.L. Rosey et al., 2010. Identification of novel pathogenicity loci in Clostridium perfringens strains that cause avian necrotic enteritis. PLOS One, 5: e10795-e10795.
Martin, T.G. and J.A. Smyth, 2009. Prevalence of netB among some clinical isolates of Clostridium perfringens from animals in the United States. Vet. Microbiol., 136: 202-205.

Petit, L., M. Gibert and M.R. Popoff, 1999. Clostridium perfringens: Toxinotype and genotype. Trends Microbiol., 7: 104-110.

Popoff, M.R. and P. Bouvet, 2009. Clostridial toxins. Future Microbiol., 4: 1021-1064.

Quinn, P.J., M.E. Carter, B.M. Maarkey and G.R. Carter, 1994. Clinical Veterinary Microbiology. Wolfe Publication Company, UK.

Rood, J.I., 1998. Virulence genes of Clostridium perfringens. Annu. Rev. Microbiol., 52: 333-360.

Smyth, J.A. and T.G. Martin, 2010. Disease producing capability of netB positive isolates of $C$. perfringens recovered from normal chickens and a cow and netB positive and negative isolates from chickens with necrotic enteritis. Vet. Microbiol.

Songer, J.G., 1996. Clostridial enteric diseases of domestic animals. Clin. Microbiol. Rev., 9: 216-234. 